\title{
Performance of MIMO-OFDM system using Linear Maximum Likelihood Alamouti Decoder
}

\author{
Monika Aggarwal $^{1}$, Suman Sharma ${ }^{2}$ \\ ${ }^{12}$ Bhai Gurdas Engineering College \\ Sangrur (Punjab) \\ monikaaggarwal 76 @yahoo. $\mathrm{com}^{1}$ \\ sumansharma2711@gmail. $\mathrm{com}^{2}$
}

\begin{abstract}
:
A MIMO-OFDM wireless communication system is a combination of MIMO and OFDM Technology. The combination of MIMO and OFDM produces a powerful technique for providing high data rates over frequency-selective fading channels. MIMO-OFDM system has been currently recognized as one of the most competitive technology for $4 G$ mobile wireless systems. MIMO-OFDM system can compensate for the lacks of MIMO systems and give play to the advantages of OFDM system.In this paper, the bit error rate (BER) performance using linear maximum likelihood alamouti combiner (LMLAC) decoding technique for space time frequency block codes(STFBC) MIMO-OFDM system with frequency offset (FO) is being evaluated to provide the system with low complexity and maximum diversity. The simulation results showed that the scheme has the ability to reduce ICI effectively with a low decoding complexity and maximum diversity in terms of bandwidth efficiency and also in the bit error rate (BER) performance especially at high signal to noise ratio.
\end{abstract}

\section{Keywords:}

Space time frequency block codes, multiple input multiple output orthogonal frequency division multiplexing,linear maximum likelihood alamouti combiners, frequency offset

\section{Introduction}

Multiple transmit and receive antennas can be used to form multiple-input multiple-output (MIMO) channels to increase the capacity by a factor of the minimum number of transmit and receive antennas. Orthogonal frequency division multiplexing OFDM is an effective technique to mitigate inter-symbol interference that multipath delay may cause in a frequency selective environment $[1,2]$. It is also evident that the combination of MIMO and OFDM produces a powerful technique for providing high data rates over frequencyselective fading channels.

For this case, MIMO-OFDM was introduced, where resulting major channel coding approach , which is space time frequency (STF) coding [3] . STFBC (space time frequency block coding) method combining ICI self-cancellation scheme and antenna, time, frequency diversity is studied to reduce ICI effectively. STFBC method using diversity technique can show excellent performance without the loss of bandwidth efficiency, compared with the other conventional methods, although it lowers the transmission speed. In space-time-frequency block coded 
orthogonal frequency division multiplexing (STFBC-OFDM), data symbols are encoded over several antennas, several symbol intervals, and several subcarriers. Thus, the influence of intercode interference (ICI) on STFBC-OFDM is smaller than that on space-time block coded OFDM (STBC-OFDM) under time selective fading and that on space-frequency block coded OFDM (SFBC-OFDM) under frequency selective fading. The first space time codes proposed by Tarokh V, Jafarkhani H, and Calderbank A.R. [4]for coherent systems over MIMO quasi static flat fading channels, by introduced their space time trellis coding technique and Alamouti introduced his STFBC techniques to improve link-level performance based on diversity [5] . OFDM has become more complex and time consuming in MIMO system utilizing maximum likelihood (ML) decoding, mainly due to the large size of the system constellation and the codeword structure and it become the second problems addressed in this paper. To overcome these problems, the LMLAC decoding techniques can be introduced as they can provide a satisfactory decoding performance in most cases and a simple method [6].In this paper the LMLAC decoding techniques is introduced in STFBC whereby to achieve low-complexity. LMLAC decoding of orthogonal space time block codes brings the complexity involved in the application of such codes to implementable levels (even with higher order modulation schemes). Purposely, this paper is focuses on the study of identifying the performance and development of STFBC MIMO-OFDM by introducing the LMLAC decoder to enable maximum diversity order against Rayleigh fading channel, to obtain highest Eb/No, lowest noise based on BER.

This paper is organized in sections as follows: In section II, STFBC in MIMO-OFDM system with FO is discussed and have derived a different LMLAC decoding. In section III, simulation results will be analyzed in terms of BER and Eb/No performance. Finally, some concluding remarks are delivered in section IV.

\section{Methodology}

\subsection{System Model}

As illustrated in Fig. 1, we focused on MIMO-OFDM system with $M=2$ Transmit and $N=2$ Receive antennas. Let the number of subcarriers in the OFDMmodulators as $K$. Using typical urban (TU) channel, the $L$-path quasi static Rayleigh fading channel model between each pair of transmit and receive antennas used in this system is six paths COST207. In the case of MIMO-OFDM, the repetition is done with $r=2$ where $r$ is how many times the data is repeated. The interference cancellation modulation (ICM) is then applied to STFBC using the repeating scheme but the repeated symbols are signed-reversed to form a new data conversion subcarrier mapping scheme ICISC technique codeword as follows: 


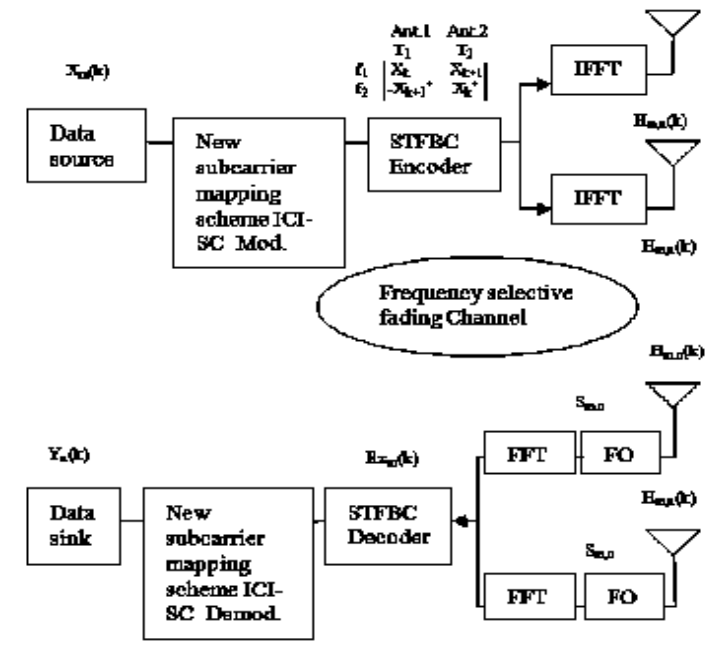

Figure 1. Block Diagram for STFBC with New Subcarrier Mapping Scheme ICI-SC Technique using a MIMO-OFDM System.

\section{Space Time Frequency Block Coding(STFBC)}

The Space Time Frequency Block Codes (STFBC) that exploit both spatial, time and frequency diversity can be designed using Orthogonal Frequency Division Multiplexing (OFDM). Intermarried Interference (ICI) self-cancellation schemes were often employed in many OFDM systems as a simple and effective approach to suppress ICI caused by Carrier Frequency error (CFO). a space time frequency block coding technique MIMO-OFDM system with ICI selfcancellation of data conjugate method, which is capable of both error correction and ICI reduction produced by Frequency Offset (FO).

Space, time, and frequency are performed using space time (ST) code and space frequency (SF) code where the same symbols are transmitted through multiple antennas at different times and frequency. The encoding of STFBC is accomplished by the following [3] as shown in Fig. 2 where Ti (time slots), $f$ (frequencies) and Ant (Antennas).

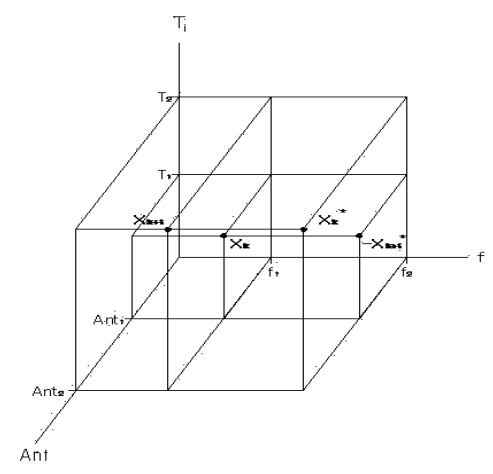

Figure 2. Coding in STFBC Method

When the channel is frequency selective, coding intelligently across the spatial, temporal and frequency domains results in a diversity order equal to the product of the channel order, the 
number of transmit and the number of receive antennas [7]. Coding for such channels is called Space-Time-Frequency Coding (STFC). In this paper, we deal with Space-Time-Frequency Block Codes (STFBCs).

Now, let the MIMO channel is assumed to be constant over each OFDM block period, but vary from one OFDM block to another (Hirosaki, B., 1981). In the same manner, the received kth subcarriers are assumed to be perfectly sampled and the received signal at the receive antenna can be expressed as follows for the MIMO systems;

$$
Y_{a}(k)=\sum_{a=1}^{M} X_{\mathbf{a}}(k) H_{a, a}(k) S_{a, a}(0)+z_{a}(k)
$$

Space, time, and frequency is performed using ST code and SF code where the same symbols are transmitted through multiple antennas at different times and frequency. The encoding of STFBC is accomplished by the following (Weifeng $\mathrm{Su}, 2005$ ) as shown in Figure 2 where Ti (time slots), $f$ (frequencies) and Ant (Antennas).

The STFBC codeword has the form of,

$$
X_{\mathrm{s}}=\left[\begin{array}{lll}
X_{1}(0) & \cdots & X_{2}(0) \\
X_{1}(0) & \cdots & -X_{2}(0) \\
\cdot & & . \\
X_{1}(k-1) & \cdots & X_{2}(k-1) \\
X_{1}(k-1) & \cdots & X_{2}(k-1)
\end{array}\right]
$$

In the case of MIMO-OFDM, the repetition is done with $r=2$ where $r$ is how many times the data is repeated. The interference cancellation modulation (ICM) is then applied to STFBC using the repeating scheme but the repeated symbols are signed-reversed to form new subcarrier mapping scheme (ICI-SC technique) codeword as followed:

$$
X_{=}=\left[\begin{array}{lll}
X_{1}(0) & \cdots & X_{2}(0) \\
-X_{1}\left(\frac{N}{2}-1\right) & \cdots & -X_{2}\left(\frac{N}{2}-1\right) \\
X_{1}\left(\frac{N}{2}-1\right) & \cdots & X_{1}\left(\frac{N}{2}-1\right) \\
-X_{1}\left(\frac{N}{2}-1\right) & \cdots & -X_{1}\left(\frac{N}{2}-1\right) \\
X_{1}\left(\frac{N}{2}\right) & \cdots & X_{2}\left(\frac{N}{2}\right) \\
-X_{1}\left((N-1)+\frac{N}{2}\right) & \cdots & -X_{2}\left((N-1)+\frac{N}{2}\right) \\
X_{1}(N-1) & \cdots & X_{2}(N-1) \\
-X_{1}\left((N-1)+\frac{N}{2}\right) & \cdots & -X_{2}\left((N-1)+\frac{N}{2}\right)
\end{array}\right]
$$


Applying the conjugate interference cancellation modulation (ICM) scheme to the repeating signal to reduce ICI, the codeword becomes:

$$
X_{2}=\left[\begin{array}{lll}
X_{1}(0) & \cdots & X_{2}(0) \\
-X_{1}\left(\frac{N}{2}-1\right)^{*} & \cdots & -X_{1}\left(\frac{N}{2}-1\right)^{*} \\
X_{1}\left(\frac{N}{2}-1\right) & \cdots & X_{2}\left(\frac{N}{2}-1\right) \\
-X_{1}\left(\frac{N}{2}-1\right)^{*} & \cdots & -X_{1}\left(\frac{N}{2}-1\right)^{*} \\
X_{1}\left(\frac{N}{2}\right) & \cdots & X_{2}\left(\frac{N}{2}\right) \\
-X_{1}\left((N-1)+\frac{N}{2}\right)^{*} & \cdots & -X_{2}\left((N-1)+\frac{N}{2}\right)^{*} \\
X_{1}(N-1) & \cdots & X_{1}(N-1) \\
-X_{1}\left((N-1)+\frac{N}{2}\right)^{*} & \cdots & -X_{1}\left((N-1)+\frac{N}{2}\right)^{*}
\end{array}\right]
$$

By allocating a pair of complex signals, the phase different between two adjacent subcarriers varies with

respect to the signal itself (Dung Ngoc Đào, 2005). This method is called new data conjugate subcarrier mapping scheme (ICI-SC technique).

In the case of MIMO-OFDM, the repetition is done with $r=2$ where $r$ is how many times the data is repeated. In the MIMO-OFDM systems, with subcarries, the coefficients from [8] is a constant with respect to subcarrier index $\mathrm{k}=0$, where is the normalized frequency offset (NFO).

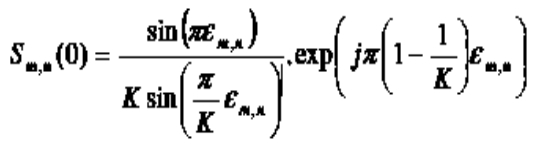

Three LMLAC decoding techniques are considered; namely the conventional combiner, maximum diversity combiner and orthogonal combiner. Different LMLAC decoding techniques have different pairs to combine the received signals and the average Eb/No with combination space time and space frequency is also different.

\subsection{Conventional combiner}

In conventional ML decoder, the received signals at times $(T)$ and frequencies $(F) k$ and $k+l$ for STFBC can be written as follows:-

$$
\begin{aligned}
& Y_{T}(k)=H_{1,1}(\mathrm{l}) S_{1}+H_{1,2}(\mathrm{l}) S_{2} \\
& Y_{T}(k+1)=H_{2,1}(2) S_{1}-H_{2,2}(2) S_{2} \\
& Y_{F}(k)=H_{1,1}(1) S_{1}+H_{2,1}(1) S_{2}
\end{aligned}
$$




$$
Y_{F}(k+1)=H_{2,2}(1) S_{1}-H_{1,2}(1) S_{2}
$$

by which the transmit symbol using maximum likelihood equations, $S 1$ and $S 2$ are given as

$$
\begin{aligned}
& S_{1}=\left(X_{1}(k)\left[H_{1,1}(k) S(0)+H_{21}(k)^{*} S(0)^{*}\right]-X_{2}(k)^{*}\left[H_{12}(k) S(I)+H_{22}(k)^{*} S(-1)^{*}\right]\right. \\
& \left.+\left(l / 4 \sum_{-0}^{N_{-1}^{-1} X_{1}(l) H_{4}(k) S(l-k)+} X_{2}(l)^{*} H_{22}(l)^{\circ} S(l-k-l)\right)+N_{2}\right) \\
& S_{2}=\left(X_{2}(k) H_{21}(k) S(0)-H_{12}(k)^{*} S(0)^{*}\right]+X_{1}(k)^{*}\left[H_{22}(k) S(1)+H_{\mathrm{u}}(k)^{*} S(-1)^{*}\right]
\end{aligned}
$$

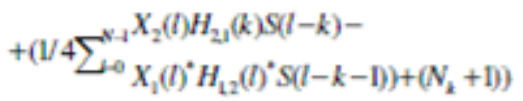

The channel coefficient is $H_{m, n}$ whereas the value of $m, n$ and $k$ are indexes of transmitting antennas, subcarriers and time. Then, the pairs $\left(H_{L, 1}^{*}(1), H_{2,1}(1)\right),\left(H_{2,1}^{*}(1),-H_{1,1}(1\right.$ and $\left(H_{1,1}^{*}(2), H_{2,1}(2)\right),\left(H_{2,1}^{*}(2),-H_{\mathrm{L}, 1}(2\right.$ that are used to combine the received signals $1 Y$ and $2 Y$ at $k$ and $k+1$ for time domain become;

$$
\begin{aligned}
& Y_{1}(k)=H_{\mathrm{u}}(1)^{*} Y_{T}(k)+H_{2,1}(1) Y_{T}(k+1)^{*} \\
& Y_{2}(k)=H_{U}(2)^{*} Y_{T}(k)+H_{2}(2) Y_{T}(k+1)^{*} \\
& Y_{i}(k+1)=H_{21}(1)^{*} Y_{T}(k)-H_{4}(1) Y_{T}(k+1)^{*} \\
& Y_{2}(k+1)=H_{21}(2)^{*} Y_{T}(k)-H_{1}(2) Y_{T}(k+1)^{*}
\end{aligned}
$$

Consequently, the frequency domain that are being used to combine the received signal are; $\quad\left(H_{L, 2}^{*}(1), H_{2,2}(1)\right),\left(H_{2,1}^{*}(1),-H_{1,2}(1)\right)_{\text {for }}$ the received signals $Y 1$ and $\left(H_{1,1}^{*}(2), H_{2,1}(2)\right),\left(H_{2,1}^{*}(2),-H_{1,1}(2)\right)$ for the received signals $Y 2$. The received signal for frequency domain becomes;

$$
\begin{aligned}
& \bar{Y}_{1}(k)=H_{\mathrm{L} 2}(1)^{*} Y_{F}(k)+H_{2,2}(1) Y_{F}(k+1)^{*} \\
& \bar{Y}_{2}(k)=H_{\mathrm{L}}(2)^{*} Y_{F}(k)+H_{2,}(2) Y_{F}(k+1)^{*} \\
& \bar{Y}_{1}(k+1)=H_{22}(1)^{*} Y_{F}(k)-H_{\mathrm{L} 2}(1) Y_{F}(k+1)^{*} \\
& \bar{Y}_{2}(k+1)=H_{21}(2)^{*} Y_{F}(k)-H_{\mathrm{L}}(2) Y_{F}(k+1)^{*}
\end{aligned}
$$

Substituting Eqs. (12), (13), (16) and (17) into Eq. (20), then the transmit symbols resulted using conventional combiner is:-

$$
\hat{S}_{1}=Y_{1}(k)+Y_{2}(k)+\bar{Y}_{1}(k)+\bar{Y}_{2}(k)
$$

and substituting Eqs. (14), (15), (18) and (19) into Eq. (21) 


$$
\hat{S}_{2}=Y_{1}(k+1)+Y_{2}(k+1)+\bar{Y}_{1}(k+1)+\bar{Y}_{2}(k+1)
$$

By referring to [19], the equation of average Eb/No via the combination of space time and space frequency it becomes;

$$
\xi=\frac{\left(1+\alpha_{i j}\right) \sigma_{H}^{2} \sigma_{s}^{2}}{2 \sigma_{*}^{2}+\left(\rho_{i}\right) \Gamma(3 / 2) \sigma_{H}^{2} \sigma_{s}^{2}}
$$

by which $\sigma_{S}^{2}$ is the average energy of the transmit symbols, $\sigma_{H}^{2}$ is the average power of the channel gain, $\sigma_{w}^{2}$ is the average noise, $\alpha_{i j}$ is the complex gain at the value of $i$ (time) and $j$ (frequency), and $\rho_{i j}$ is the normalized interference coefficient transmit symbols at $i$ and $j$.

\subsection{Maximum diversity combiner}

We now propose a second technique to achieve performance of low complexity, with maximum diversity order. By using the same mapping method, the received signal for time domain pair are $\left(H_{2,2}^{*}(1), H_{2,2}(2)\right),\left(H_{2,2}^{*}(2),-H_{1,2}(2)\right.$ and $\left(H_{1,1}^{*}(1), H_{2,1}(2)\right),\left(H_{2,1}^{*}(1),-H_{2,1}(2)\right)$, and for frequency domain pair are $\left(H_{1,1}^{*}(1), H_{2,2}(1)\right),\left(H_{2,1}^{*}(1),-H_{1,2}(1)\right)$ and $\left(H_{1,1}^{*}(2), H_{2,2}(2)\right)$, $\left(H_{21}^{*}(2),-H_{12}(2)\right)$.

By employing the same method as in Eqs. (17) and (18) on different pairs, we obtained the equations $\hat{S}_{1}$ and $\hat{S}_{2}$. From [16], the resulting average Eb/No for space time and space frequency is;

$$
\xi=\frac{2 \sigma_{H}^{2} \sigma_{S}^{2}}{2 \sigma_{w}^{2}+\left(\rho_{i j}\right) \sigma_{H}^{2} \sigma_{S}^{2}}
$$

\subsection{Orthogonal combiner}

Next, we propose another technique known as orthogonal combiner. Let's substitute Eqs. (9) to (16) into the equations for $\hat{S}_{1}$ and $\hat{S}_{2}$, then, combine

different pairs in the time domain $Y_{1}=\left(H_{\mathrm{L}, 1}^{*}(2), H_{2,1}(1)\right), \quad\left(H_{2,1}^{*}(2),-H_{L, 1}(1)\right.$ and $Y_{2}=\left(H_{1,2}^{*}(2), H_{2,2}(1)\right),\left(H_{2,2}^{*}(2),-H_{1,2}(1)\right)$ and apply the frequency domain $Y_{1}=\left(H_{1,2}^{*}(1), H_{1,1}(1)\right)$, $\left(H_{2,2}^{*}(1),-H_{1,1}(1)\right)$ and $Y_{2}=\left(H_{1,2}^{*}(2), H_{2,1}(2)\right),\left(H_{2,2}^{*}(2),-H_{1,1}(2)\right)$.

From the above pairs, the received signals for orthogonal combiner can obtain $\hat{S}_{1}$ and $\hat{S}_{2}$ respectively. The average Eb/No with the combination of space time and space frequency is as obtained below [8];

$$
\xi=\frac{2\left(\alpha_{i j}\right) \sigma_{H}^{2} \sigma_{S}^{2}}{2 \sigma_{w}^{2}}
$$

At this stage, we can conclude that, in STFBC MIMO-OFDM, as the average Eb/No becomes higher, the noise in system become lower. 


\section{Simulation Results and Discussion}

This section shows the simulation of the proposed STFBC design methods with the insertion of LMLAC Decoding. For this system, we use the six-path COST 207 (Jakes model) typical urban (TU) channel model [18] over a more realistic model. By using OFDM base, the simulation parameters are shown in Table 1.

Table 1. Simulation Parameters for the System [10].

\begin{tabular}{ll}
\hline Parameters & \multicolumn{1}{c}{ Value } \\
\hline Bandwidth (BW) & $1.25 \mathrm{MHz}$ \\
Sampling frequency & $1.92 \mathrm{MHz}$ \\
Sampling time & $5.208 \times 10^{-7}$ second \\
No. of subcarriers & $76 \mathrm{subcarriers}$ \\
Modulation technique & $64-\mathrm{QAM}$ \\
$\begin{array}{l}\text { Maximum Doppler } \\
\text { frequency }\end{array}$ & $120 \mathrm{~Hz}$ \\
IFFT size & 128 \\
Channel model & COST207 Typical Urban $(\mathrm{TU})$ channel \\
& Path delays, $L_{p}=\left(0,0.2 \times 10^{-6}, 0.5 \times 10^{-6}\right.$, \\
& $\left.1.6 \times 10^{-6}, 2.3 \times 10^{-6}, 5.0 \times 10^{-6}\right)$ seconds \\
& Average path gains $=[0.5011,1.122$, \\
& $0.6309,0.251,0.158,0.1] \mathrm{dB}$ \\
\hline
\end{tabular}

The simulation results presented BER curves as functions of Eb/No as shown in Fig. 3. Figure 3, depicts the BER performance of STFBC with new data conjugate and data conversion subcarrier mapping scheme ICI-SC technique by using four types of subcarrier mapping compared at $\mathrm{NFO}=5 \%$. BER for data conjugate improves at high Eb/No than conversion method for all decoding methods using new subcarrier mapping scheme. The data conjugate with maximum diversity combiner has the best BER performance compared to the other three decoding techniques because the system has a low decoding complexity system with optimal distance that can achieve maximum frequency diversity and less interference. For instance, at $\mathrm{BER}=2 \times 10-2$, the $E b / N o$ value for maximum diversity combiner with data conjugate is 4.4 $\mathrm{dB}$. The performance loss of data conjugate between maximum diversity combiner with conventional combiner, orthogonal combiner and ML decoding are about $2.1 \mathrm{~dB}, 4 \mathrm{~dB}$ and $4.5 \mathrm{~dB}$ respectively. It is noticeable that the ML decoding performed worse than other techniques in linear combiner and performs the lowest value of Eb/No. It is also shown that maximum diversity combined with new data conjugate subcarrier mapping scheme ICI-SC technique from Eq. (2) yields the best performance with low complexity and less time consumption compared with other linear combiner techniques, and produces the highest Eb/No values from Eq. (20). In overall, linear combiner performs at better BER performance with higher $E b / N o$ from Eqs. (19), (20) and (21) than the ML decoding with a NFO=5\%. 


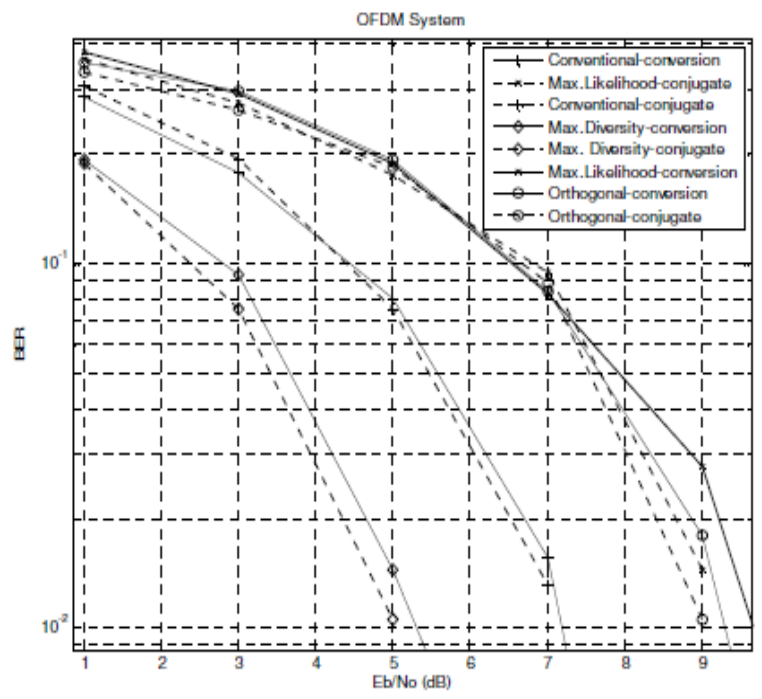

Figure 3. STFBC Systems for $f o=0.05$ with New Data Conjugate and Data Conversion Subcarrier Mapping Scheme ICI-SC Technique Using Different Decoding Techniques.

Figure 4 depicts the simulation results of BER performance of STFBC with new data conjugate subcarrier mapping schemes ICI-SC technique with $\mathrm{NFO}=(0 \%, 5 \%, 15 \%, 20 \%)$ using maximum diversity combiner decoding technique. Figure 4 shows that when the NFO is $0 \%$ at $\mathrm{BER}=3 \times 10-2$, the performance loss is about $0.9 \mathrm{~dB}$ for $\mathrm{NFO}=5 \%, 2.2 \mathrm{~dB}$ for $\mathrm{NFO}=15 \%$ and $6 \mathrm{~dB}$ for $\mathrm{NFO}=20 \%$ respectively. The value of $E b / N o$ needs to be compensated for the effect of $\mathrm{FO}$ increases. The new data conjugate subcarrier mapping scheme from Eq. (2) at $\mathrm{NFO}=0 \%$ produces the best BER performance for all NFO in the system. It is proven that there is a significant improvement when the value of FO decreases in the system. The above simulation shows that the lower the FO the better performance of the system; which can increase the Eb/No and decrease the BER. If the FO decreases, the shift of BER curves with higher diversity order is larger than the shift of BER curves with lower diversity order. Therefore, the higher diversity order systems are more robust to the effect of FO. From the above simulation, the result confirm that the BER performance for a new subcarrier mapping scheme ICI-SC technique using maximum diversity combiner method produces ICI reduction in the system with low complexity decoding technique and can achieve maximum diversity order. 


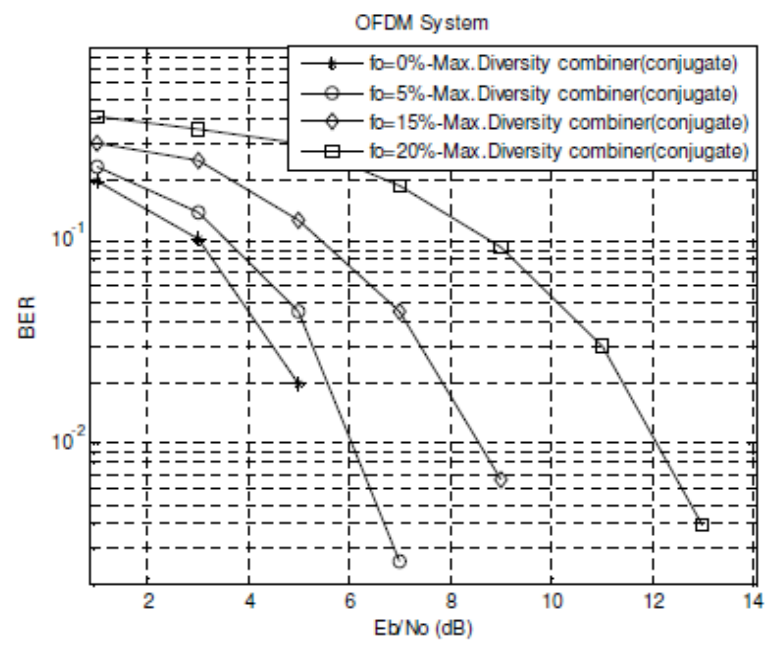

Figure 4. STFBC Systems for Different fo with New Data Conjugate Subcarrier Mapping Scheme ICI-SC Technique Using Maximum Diversity Combiner Decoding Techniques.

\section{Conclusions}

In this paper, a new subcarrier mapping technique that is combined with LMLAC decoding techniques is proposed using STFBC MIMO-OFDM system. The simulation results have shown that the performance improvement of BER can achieve the objectives of this paper to study for ICI reduction methods with low decoder complexity and maximum diversity order system. These has proven that the proposed subcarrier mapping ICI-SC technique combined with maximum diversity combiner technique using STFBC can be considered as a promising candidate in the MIMO OFDM based system.

\section{REFERENCES}

[1] Falconer, D.; Ariyavisitakul, S.L.; Benyamin-Seeyar, A.; and Eidson, B. (2002). Frequency domain equalization for single-carrier broadband wireless systems. IEEE Communication Magazine, 40(4), 58-66.

[2] Omar, M.H.; Hassan, S.; and Shabli, A.H.M. (2009). COGRADNET: Ubiquitous heterogeneous wireless networks. Journal of Engineering Science and Technology (JESTEC), 4(2), 184-195.

[3] Weifeng Su, Zoltan Safar and K.J. Ray Liu, "Towarda Maximum Achievable Diversity in Space, Time , and Frequency: Performance Analysis and Code Design," IEEE Trans On Wireless Communications, vol. 4, pp 1847-1857, July 2005.

[4] Tarokh V , Jafarkhani H and Caklerbank AR.,"Space-time block coding for wireless communications:performance results,"IEEE Journal on Selected Areas in Communications, Vol.17(3), pp.451-460, 1999.

[5] Alamouti, Siavash, "A Simple Transmit Diversity Technique for Wireless Communications," IEEE Journal on Selected Areas in Communications, Vol. 16, pp. 1451-1458.October 1998.

[6] de Abreu, G.T.F.; Ochiai, H.; and Kohno, R.. (2004). Linear maximum likelihood decoding of space-time block coded OFDM systems for mobile communications. IEE ProceedingsCommunications, 151(5),447-459. 
[7] Z. Liu, Y. Xin, and G. B. Giannakis, "Space-Time-Frequency Coded OFDM over Frequencyselective fading channels," in IEEE Trans. Signal processing, vol. 50, no. 10 pp. 2465-2476, October 2002.

[8] Y.Zhao and S-G Haggman, "Intercarrier interference self cancellation scheme for OFDM mobile communication systems," IEEE Transactions on Communications, Vol. 49, pp. 1185-1191,2001.

[9]. de Abreu, G.T.F.; Ochiai, H.; and Kohno, R.. (2004). Linear maximum likelihood decoding of space-time block coded OFDM systems for mobile communications. IEE ProceedingsCommunications, 151(5),447-459.

[10]. Chiueh, T.D.; and Tsai, P.Y. (2007). OFDM baseband receiver design for wireless communications. John Wiley and Sons (Asia) Pte Ltd, Singapore.

\section{Authors}

Monika Aggarwal received her B. Tech. degree in Electronics and Communication engineering from SLIET(PTU), Longowal, Punjab, in 2000 and her M.Tech degree in Electronics and Communication engineering from Punjab Technical University, Jalandhar, Punjab, in 2009. She is currently working as Associate Professor at Bhai Gurdas Institute of Engineering and Technology, Sangrur, Punjab, India .

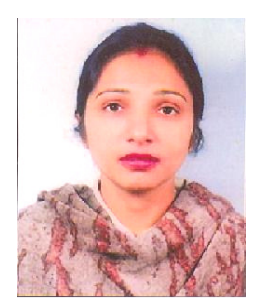

Suman Sharma received her B. Tech degree in Electronics and Communication engineering from MMEC(KU), Mullana, Haryana, in 2008. She is currently persuing her M.Tech from Bhai Gurdas Institute of Engineering and Technology, Sangrur, Punjab, India and doing her dissertation in Wireless MIMOOFDM system. 